\title{
On the occasion of publication of the feature on echocardiographic management of valvular heart disease
}

Yutaka Otsuji ${ }^{1}$

(c) The Japan Society of Ultrasonics in Medicine 2019

There are two major shifts occurring in the area of valvular heart disease. The first is a shift in valve disease itself, and the second is a shift in diagnosis and treatment. The most common valve disease when I was a resident in the early 1980s was rheumatic mitral stenosis. The number of cases of rheumatic mitral stenosis subsequently decreased dramatically with advances in the diagnosis and treatment of acute rheumatic fever. I would be willing to bet that several patients at the most undergo surgery for rheumatic mitral stenosis annually even at relatively large hospitals today. With the aging of society, we are seeing an increase in patients with degenerative aortic stenosis, prolapsed mitral valve, and ischemic and functional mitral regurgitation. And judging from the number of patients undergoing heart surgery, the number of cases of valve disease is increasing. Thus, the types of valve disease we encounter are shifting, and the total number of cases is on the rise.

Diagnostic approaches and the goals of diagnosis have also changed. The most common diagnostic approaches for valve disease in the 1980s were catheter-based diagnosis and cineangiography. In the case of mitral regurgitation, for example, the most reliable approaches were to determine the degree of staining of the left ventricle and left atrium (Sellers' classification) after insertion of a catheter into the left ventricle and injection of a contrast agent, or to evaluate the left atrial/left ventricular pressure gradient or severity of mitral stenosis based on the difference between pulmonary artery wedge pressure and left ventricular diastolic pressure using right and left heart catheterization. Yet today, I do not think there are any medical institutions that use catheters for the diagnosis of valve disease. The reason for this is the

Yutaka Otsuji

otsujiy@med.uoeh-u.ac.jp

1 Second Department of Internal Medicine, University of Occupational and Environmental Health, Kitakyushu, Japan development of noninvasive diagnostic imaging including echocardiography.

Treatment of valve disease is also shifting, and diagnostic requirements are changing. In the 1980 s, surgical valve replacement was the only fundamental therapy available. As such, the only requirement for diagnosis was to identify the severity of valve disease. In the case of mitral regurgitation, the only requirement was to determine the severity of regurgitation, as represented by Sellers' classification. However, surgical valvuloplasty and catheter-based treatments (e.g., TAVI, MitraClip) subsequently became available. Therefore, determining whether surgical valvuloplasty is possible or whether the valve disease is more suited to catheterization is also required for diagnosis today, in addition to identifying the severity of the valve disease. Consequently, the anatomical structure of the valve and the properties of the tissue, in addition to valve function, are now required for diagnosis, and providing information related to selection of the optimal treatment is required. Moreover, exercise hemodynamics in patients with valve disease is now evaluated using exercise echocardiography, and detailed assessment by intraoperative transesophageal echocardiography and postoperative echography is required. Going forward, new catheter-based treatments will become available, and surgical approaches will continue to evolve (e.g., MICS). The requirements for ultrasound diagnosis of valve disease will very likely continue to change in the future.

For this feature, we asked Dr. Nobuyuki Kagiyama, Dr. Takeshi Kitai, and Dr. Kenya Kusunose, who are young ultrasound diagnosticians/researchers in Japan, to write review articles with the latest information about ultrasound diagnosis of valve disease, which continues to change, as described above. I hope this feature will help you all in your medical practice, and that it will lead to new advances in research and diagnosis. 